\title{
The Relationship Between Cognitive Appraisal, Affect, and Catastrophizing in Patients With Chronic Pain
}

\author{
David A. Jones, ${ }^{*}$, Gary B. Rollman, * Kevin P. White, ${ }^{\dagger}, \|$ Marilyn L. Hill, $*, \uparrow, * *$ and \\ Ralph I. Brooke
}

\begin{abstract}
A study was conducted to clarify the nature of catastrophizing, a construct that is frequently referred to in the chronic pain literature. Information regarding 3 affective experience and 3 affect regulation dimensions was gathered from a heterogeneous sample of 104 chronic pain patients by using a semistructured clinical interview and the Affect Regulation and Experience Q-Sort (AREQ). Self-report questionnaires included visual analog pain scales, the Coping Strategies Questionnaire (CSQ), Multidimensional Pain Inventory (MPI), McGill Pain Questionnaire (MPQ), and Center for Epidemiological Studies Depression scale (CES-D). Hierarchical multiple regression was used to demonstrate the relative contributions of affective and cognitive appraisal components of catastrophizing. Thirty-one percent of the variance in CSQ-Catastrophizing scores was explained by a combination of cognitive appraisal variables (perceived ability to control pain; MPI Life Control) and AREQ scores, even after adjusting for pain severity and chronicity, age, and sex of participants. Results of the study strongly suggest that, rather than thinking of catastrophizing primarily as a cognitive coping construct, it should be described as an elaborate construct made up of both cognitive appraisal and affective components. Implications for tailoring interventions to match individual styles of affect regulation are discussed.
\end{abstract}

(C) 2003 by the American Pain Society

Key words: Catastrophizing, pain, coping, affect, cognitions.

I n our attempts to find factors that affect outcomes in different populations of patients with chronic pain, the concept of catastrophizing (CAT) has been identified as an important predictor of the ability to function with chronic pain. The review by Geisser et $\mathrm{al}^{9}$ of the literature concluded that maladaptive pain beliefs such as CAT are more important determinants of chronic pain adjustment than more adaptive pain coping and beliefs. Robinson et $\mathrm{al}^{30}$ demonstrated that CAT showed significant negative relationships with general activity levels and perceived life control, as well as positive relationships with reports of pain severity, perceived life interference, and levels of affective distress. Martin et $\mathrm{al}^{22}$ also demonstrated a positive relationship between CAT and both physical and psychosocial disability, noting that these relationships were dependent on scores for neu-

Received August 26, 2002; Revised March 5, 2003; Accepted March 5, 2003.

From the *Department of Psychology, Social Science Centre, ${ }^{\dagger}$ Department of Medicine, ${ }^{*}$ School of Dentistry, Faculty of Medicine and Dentistry, University of Western Ontario, London, Ontario, §Pierce Health Services, Kitchener, Ontario, "Department of Epidemiology and Biostatistics and "Arthritis Institute, St Joseph's Health Care, London, Ontario, and ** Lawson Research Institute, London, Ontario, Canada.

Supported in part by grants from the Arthritis Foundation (K.P.W.) and from the Natural Sciences and Engineering Research Council of Canada (G.B.R.).

Address reprint requests to David Jones, DMD, PhD, 411-564 Belmont Ave W, Kitchener, Ontario N2M 5N6, Canada. E-mail: jones4783@rogers.com (C) 2003 by the American Pain Society

$1526-5900 / 2003 \$ 30.00+0$

doi:10.1016/S1526-5900(03)00630-8 roticism. Martin and Bradley ${ }^{22 a}$ have also shown that these relationships appear to be similar across samples of patients with fibromyalgia, rheumatoid arthritis, and osteoarthritis. Extensive research evidence exists to support relationships between CAT and psychological distress, ${ }^{7,8,27,46}$ physical functioning and disability, ${ }^{13,22}$ ratings of pain intensity, ${ }^{13,20,22,36}$ interference with life activities, $^{7}$ and psychosocial dysfunction. ${ }^{13,15}$

Despite the growing body of research evidence documenting relationships between CAT and a number of different pain outcome measures, there is a continuing debate about the nature of CAT as a psychological construct. In everyday language, the term catastrophe is applied to people who have experienced sudden or extreme disasters or misfortune in their lives. These people become overwhelmed by the uncontrollability of their situations and by a wide variety of intense emotions such as grief, shock, despair, fear, anger, and much more. The onset and continuing experience of chronic pain are often perceived as a highly emotional and catastrophic experience by those suffering from it. However, research with chronic pain patients has tended to define CAT as a cognitive coping strategy, a set of maladaptive beliefs about one's pain, or a symptom of depression. For those who adopted the position that CAT is primarily a cognitive construct, there has been a tendency to think of affect or emotions as something that needs to be factored out of data analyses to clarify the nature of CAT. 


\section{Catastrophizing as a Coping Strategy, Appraisal, or Belief}

In their initial description of the Coping Strategies Questionnaire (CSQ), Rosenstiel and Keefe ${ }^{32}$ described CAT as 1 of 6 cognitive coping strategies that emerged from their factor analysis of items describing coping in patients with low back pain. More recently, Keefe et al ${ }^{17}$ proposed that when patients catastrophize, they are actively communicating their distress and their inability to deal with their pain as a way of coping, and that these coping attempts fit the definition of coping strategies according to the transactional coping model proposed by Lazarus and Folkman. ${ }^{19}$ However, rather than seeing CAT as actual attempts at coping, other authors have conceptualized CAT either as a form of cognitive appraisal within the transactional model ${ }^{2,40}$ or as maladaptive beliefs held by patients about their ability to cope with pain. ${ }^{9,10,15}$

This latter view of CAT as a form of cognitive appraisal is consistent with the results of the original description by Rosenstiel and Keefe ${ }^{32}$ of the CSQ subscales, which showed that the 2 individual CSQ items that measure perceived efficacy at controlling or decreasing pain both loaded heavily on a common factor with the CAT scale, indicating that these scores are highly correlated. Härkäpää ${ }^{12}$ also showed that weak beliefs in personal control over health were significantly related to CAT in patients with back pain, whereas Keefe et al ${ }^{16}$ showed that CAT was related to lower ratings of patients' selfefficacy as rated by their spouses. Thus, there is ample evidence in the literature that beliefs or appraisals regarding the inability to control or decrease one's pain play a significant role when patients are catastrophizing about their pain.

In their review of theoretical perspectives on catastrophizing, Sullivan et al $^{38}$ described the cognitive appraisal models noted above but also discussed 3 less frequently cited models: a schema activation model, an attention model, and a communal coping model. They proposed that the cognitive models of catastrophizing (schema activation and appraisal) might provide more proximal explanations or mechanisms for the relationship between catastrophizing and pain outcomes. However, they also proposed that the communal coping model might help to link social and behavioral factors to catastrophizing and provide a more distal explanation of the construct.

\section{Catastrophizing as an Emotional Experience}

Despite the observation that the intense emotional responses of victims are an integral part of the response to a catastrophe, Geisser et $\mathrm{al}^{9,10}$ have downplayed the role of affect in their previously cited models of catastrophizing and coping with chronic pain. In fact, a number of studies noted above have removed the variance attributable to negative affect from their analyses, presumably under the assumption that it is not an integral part of the catastrophizing construct. This approach implicitly reflects a belief expressed by Watson and Pennebaker ${ }^{48}$ that negative affect is a general nuisance factor in health research. However, rather than treating the negative affect associated with CAT as a nuisance factor, there is sufficient research evidence to consider the possibility that CAT is composed of affective components in addition to the beliefs or cognitive appraisal components discussed above.

A review of the literature shows a consistent relationship between high CAT scores and high scores on a number of measures of negative affect. ${ }^{15,31,35}$ A recent study by Turner et $\mathrm{al}^{44}$ showed that CAT scores are significant predictors of depression scores, even after controlling for pain beliefs and coping strategies. Those results suggest that there is unique variance in CAT that exists above and beyond both pain beliefs and coping strategies, and that this variance has a very strong relationship with affective distress. Sullivan et $\mathrm{al}^{37}$ examined the extent that measures of depression, anxiety, and CAT, as measured by their Pain Catastrophizing Scale (PCS), were able to predict disability scores. They reported that CAT, depression, and anxiety scores together accounted for $37 \%$ of the variance in disability scores, but that only CAT contributed significant unique variance. Relationships between CAT and other indicators of negative affect such as neuroticism ${ }^{1,22}$ and various types of anxiety ${ }^{4,23,47}$ have also been demonstrated. Vlaeyen and Linton ${ }^{47}$ have proposed a model detailing a fear avoidance cycle that suggests that negative affect and previous pain experiences interact through CAT to increase pain-related fear and eventually to increase pain-related disability.

Geisser et $\mathrm{al}^{8}$ used path analysis to test the hypothesis that CAT mediates the relationship between depression and the evaluative and affective aspects of pain. Results showed a significant prediction of evaluative and affective pain ratings from the McGill Pain Questionnaire (MPQ) after controlling for levels of depressive symptoms, which the authors thought demonstrated that CAT is a unique construct rather than being conceptually related to symptoms of depression. Alternatively, CAT might be a combination of both affective and cognitive/ evaluative components. By controlling for depressive symptoms, Geisser et al might simply have removed the affective component, leaving behind the cognitive component, which is predictive of pain reports. However, regardless of the interpretation, Geisser et al provided further evidence that variance due to negative affect is a significant part of CAT and plays a significant role in predicting pain outcomes as well.

Research on CAT in non-pain populations has shown that catastrophic worrying represents an internal dialogue style characterized by 4 types of internal statements: (1) problem-specific pessimism, (2) personal inadequacy/incompetence, (3) personal despair/ hopelessness, and (4) need to analyze the problem. ${ }^{6}$ These 4 categories of internal dialogue are interesting because they seem to encompass both cognitive and affective domains. Evidence for these types of internal dialogue in CAT was presented by Davey et al, ${ }^{5}$ who demonstrated that catastrophic worrying is the result of 
ongoing but thwarted coping processes. These observations are consistent with the aforementioned model of fear avoidance proposed by Vlaeyen and Linton ${ }^{47}$ for pain patients, in which negative cognitive appraisals about previous pain experiences and unsuccessful coping attempts combined with negative affect tend to be associated with CAT, leading to a fear of attempting adaptive coping strategies and an ongoing cycle of despair and disability.

The 4 types of internal dialogue proposed by Davey and Levy ${ }^{6}$ also show conceptual similarities to the components of CAT identified by Sullivan et $\mathrm{al}^{36}$ in the PCS. The 3 CAT subcomponents identified by Sullivan et al were (1) helplessness, (2) rumination, and (3) magnification. Sullivan et al demonstrated significant correlations between depressive symptoms, anxiety, negative affect, and fear of pain in their development of the PCS, which again suggested that there is a significant affective component associated with CAT. These associations were confirmed in a factor analysis of the PCS by Osman et al. ${ }^{26}$

Sullivan et $\mathrm{al}^{36}$ considered only the unique variance attributable to CAT and affective variables when predicting pain, concluding that there is no conceptual confound between CAT and depression, anxiety, negative affect, or fear of pain. In a subsequent study, Sullivan et $\mathrm{al}^{37}$ showed that the unique variance associated with the 3 PCS subscales accounted for $4 \%$ of the variance in pain disability scores, whereas the PCS together with unique variance from depression and anxiety accounted for $37 \%$ variance in disability scores. However, the approach used by Sullivan et $\mathrm{al}^{37}$ and also by Geisser and Roth, ${ }^{10}$ in which only variance in CAT that is unique from negative affect is used in data analysis, minimizes the importance of the affective experience and methods of affect regulation used by patients with chronic pain when they attempt to cope with their symptoms. The implication of these results for treating patients is that we should be considering negative affect as an integral component of CAT when considering the relationships between CAT and pain outcomes, rather than considering only the nonaffective, cognitive aspects of CAT such as pain-related beliefs or secondary appraisal. In effect, our interventions would become emotion focused as well as cognitive and behavioral.

\section{Goals of the Present Study}

A better understanding of CAT is important in increasing our knowledge of the pain experience. ${ }^{8}$ More specifically, we hypothesize that knowledge about a patient's affective experience and habitual ways of regulating affect should allow us to include treatment modules for clients that would address not only their cognitions and beliefs but would also focus on improving deficits in affect regulation that often interfere with the client's ability to manage their pain. Consequently, the goal of this study was to focus on the nature of the catastrophizing construct itself to better comprehend what is happening when a patient with chronic pain is catastrophizing and consequently to improve our ability to explain relation- ships between CAT and pain outcome variables. The tested hypothesis was that CAT is a complex construct composed of both secondary appraisal components and affective components. Specifically, it was proposed that CSQ scores on the CAT scale would be predicted by the specific affective experience and regulation factors measured by the Affect Regulation and Experience Q-Sort (AREQ), ${ }^{49}$ as well as by variables measuring secondary cognitive appraisal from the CSQ and the Multidimensional Pain Inventory. ${ }^{18}$

\section{Materials and Methods}

\section{Participants}

To maximize the generalizability of our results, we selected chronic pain patients with a broad range of diagnoses, including rheumatoid arthritis (RA) and osteoarthritis (OA), fibromyalgia syndrome (FMS), temporomandibular dysfunction (TMD), or various types of localized chronic pain. No intergroup comparisons were performed because a growing body of literature has demonstrated that there is greater variance in psychological variables within than between diagnostic groups. ${ }^{29,33,34}$ We also permitted subjects with multiple diagnoses because we believed that having more than one diagnosis might be relevant to the emotional experience of chronic pain.

Our study was cross-sectional in design. All participants were adults older than the age of 18 years. Five distinct chronic pain populations were identified and recruited: (1) individuals with TMD who had not experienced a traumatic joint injury but might have exhibited muscular pain, disk displacements, OA, or OA of the temporomandibular joint; (2) individuals with chronic pain localized to the back, neck, or limbs; (3) individuals with chronic generalized pain who met the 1990 American College of Rheumatology (ACR) classification criteria for FMS and had been referred to a rheumatology outpatient clinic; (4) individuals with chronic generalized pain who had been recruited as part of a community survey and were found to meet the ACR definition for FMS ${ }^{50}$; and (5) individuals with RA or OA who had been referred to an outpatient rheumatology clinic.

Participants with a single pain diagnosis of TMD pain $(\mathrm{N}=18), \mathrm{FMS}(\mathrm{N}=20)$, RA or OA $(\mathrm{N}=8)$, or pain localized to the back, neck, or limbs $(N=2)$ comprised $54 \%$ of those patients who had been previously diagnosed with a pain disorder and $46 \%$ of the overall sample when the community sample of FMS patients is considered. Participants with 2 or more of the various pain diagnoses mentioned above comprised $46 \%$ of previously diagnosed patients and $39 \%$ of the overall sample. The community FMS sample $(\mathrm{N}=15)$ made up $14 \%$ of the overall sample.

Potentially eligible patients were identified by a review of all office charts for patients with any of the previously listed diagnoses who had been assessed by the participating rheumatologist (K.P.W.) or dentist (R.I.B.) within the previous year, and who had been experiencing their pain for a period of at least 6 months. Eligible participants received a letter of information explaining 
the general nature of the study, and individual appointments were arranged for people who were interested in participating. Potential participants were included in the study if they were proficient enough in English to be interviewed and understand the questionnaires. Patients were not excluded if they had participated in a pain management program or if they were receiving active treatment (ie, ongoing physiotherapy, medication, or psychotherapy). They were excluded if they were currently experiencing psychotic symptoms that would have affected their ability to provide a valid clinical interview. Twenty-one percent of eligible patients $(N=104)$ responded to their letter and met the criteria for the study. This group (94 women and 10 men) had a mean age of 45.51 years (standard deviation, 11.39). This study was approved by The University of Western Ontario Review Board for Health Sciences Research Involving Human Subjects.

\section{Procedure}

The study was explained to each participant and informed consent was obtained. Each participant initially completed a brief introductory questionnaire that asked about age, sex, pain diagnoses, pain chronicity, current treatment status, participation in pain treatment programs, mental health history, and involvement in litigation or compensation disputes. Participants then engaged in a semistructured clinical interview, the Affect Regulation Interview (ARI). ${ }^{49}$ The interviews were conducted by the first author (D.A.J.) and required approximately 60 to 75 minutes to administer. The interviews were followed by administration of questionnaires containing a battery of standardized psychometric measures. Videotapes of the interviews were kept as a backup in case clinical observations from the interviews could not be quantified by using a Q-sort procedure within 12 hours of the interview. A random sample of 12 interview tapes $(10 \%$ of the interviews in this and a pilot study) were rated by a second observer (M.L.H.) to assess interrater reliability of the Q-sorting procedure, and a random sample of 10 interview tapes were rated again by the first observer after a period of 2 to 4 weeks and a number of intervening interviews had elapsed to calculate test-retest reliability. Both raters trained by using interviews from the pilot study to assure maximum adherence to the instructions provided by the authors of the AREQ and ARI and to maximize interrater reliability.

The AREQ was used to quantify clinical observations from the ARI. ${ }^{49}$ The 98 AREQ items were typed onto individual cards that were initially sorted into 3 stacks of 48,20 , and 30 cards labeled "not descriptive," "very descriptive," or "ambiguous," respectively, according to how well they described the participant. A second round of sorting divided the 3 original stacks into 3 more stacks for a total of 9 stacks containing 22, 16, 10, 10, 10, 10, 10, 6 , and 4 cards. The 22-card stack contained items that were least descriptive of the participant, and the 4-card stack contained cards that best described that person. Cards in each stack were assigned scores ranging from 1 (for the 22-card stack of nondescriptive items) to 9 (for the 4-card stack of very descriptive items). Thus, each AREQ item was assigned a score ranging from 1 to 9 on a ordinal scale, in which a score of 9 indicated items that were most descriptive of that person and a score of 1 indicated items that were least descriptive. Items with scores in the middle range were items that were only somewhat descriptive or ambiguous descriptors of that person.

\section{Psychometric Measures}

\section{ARI}

The ARI is a semistructured interview that was used to collect information about affective experiences and methods of affect regulation and provided the basis for the AREQ. The ARI was modified slightly for use with patients with chronic pain and to ensure that information pertaining to all AREQ items was collected during the interview. The ARI asks participants to provide information about current life stressors (including but not limited to chronic pain), history of relationships (with family, friends, partners, and coworkers), activities, coping styles, and about how each individual tended to experience and regulate emotions in the different areas of their lives. The ARI is organized such that it gathers information related to the 98 items of the AREQ, thus providing the information essential for performing the Qsort procedure.

\section{AREQ Q-Sort}

The 98 items comprising the AREQ are behavioral descriptions of ways that people commonly experience or regulate emotions. Two separate factor analyses of the items that describe either affective experience (AE) or affect regulation (AR) have been performed by Westen et al, ${ }^{49}$ both resulting in solutions with 3 bipolar dimensions that have good convergent and discriminant validity, as well as good predictive validity. They report interrater reliability of $r=.78$ for the AREQ. The $3 \mathrm{AE}$ dimensions were described by the authors as Socialized Negative Affect, Positive Affect, and Intense Negative Affect. The 3 AR dimensions were described as RealityFocused Coping, Externalizing Defenses, and Avoidant Defenses.

The extraction of 3 AE dimensions, including 2 different negative affective dimensions, differs from most existing research, which has often described only a single positive and a single negative affective dimension. The Socialized Negative Affect dimension describes emotions that require internalization of social norms and values (such as shame, guilt, regret, and embarrassment) at its positive pole. At its negative pole, Socialized Negative Affect items describe a failure to internalize social norms, an inability to feel the internalized emotions indicated above, and a lack of empathy. In contrast, the positive pole of the Intense Negative Affect dimension describes people who are easily overwhelmed by emotions such as anxiety or depression, whereas the negative pole describes people who have poor ability to be aware of, identify, and label emotions. 


\section{Self-Report Questionnaires}

Body Map Questionnaire (BMQ). ${ }^{24}$ Patients indicated on separate diagrams representing the front and back of their body all areas in which they were currently experiencing pain symptoms. Patients rated pain severity at each reported pain site by using $100-\mathrm{mm}$ visual analog scales (VAS). Pain severity was calculated as the mean VAS pain ratings for all pain sites reported by each participant.

The Coping Strategies Questionnaire (CSQ). ${ }^{32}$ This 44-item self-report inventory was designed to assess 6 cognitive and behavioral dimensions used to cope with chronic pain by using a Likert scale ranging from 0 (never used) to 6 (almost always used). The present study used only the catastrophizing subscale and 2 items that asked respondents to use 7-point rating scales to appraise their perceived extent of control over their pain and their ability to decrease pain. The CSQ was given preference over the more recently developed PCS ${ }^{36}$ because the CSQ catastrophizing scale has been shown by a number of studies to be a stable global measure of the construct, as noted below. Furthermore, the 2 cognitive appraisal items (\#43 and \#44) from the CSQ, which measure perceived abilities to decrease or control pain, were required because they were an essential part of the hypothesis for this study. The CSQ has demonstrated good internal consistency (alpha coefficients ranging from 0.72 to 0.85 ) and acceptable test-retest reliability (correlations ranging from $r=0.88$ to 0.93 ). ${ }^{21}$ Two separate factor-analytic studies ${ }^{39,45}$ have demonstrated 5 stable subscales, compared to the 6 proposed by Rosenstiel and Keefe. The catastrophizing subscale has emerged as one of these stable factors in most factoranalytic studies of the CSQ. However, as noted in the introduction, the nature of the catastrophizing construct has been an ongoing research question and provides the basis for the present study.

Multidimensional Pain Inventory (MPI). ${ }^{18,42,43}$ Part 1 of the MPI assesses the psychosocial impact of chronic pain on a patient's life, including measures of pain severity, life interference, life control, affective distress, and social support. For the present study, 18 items from the life interference, life control, and social support scales were administered. Part 2 assesses the patient's behavioral responses to others and was not administered in this study. Part 3 measures level of daily activity in the areas of household chores, outdoor work, activities away from home, social activities, and general activity level. All 18 items from part 3 were administered, and the aggregate score for general activity level was used in subsequent statistical analyses. All items were answered on Likert scales ranging from 0 (no endorsement) to 6 (extreme endorsement). Internal consistency of MPI subscales ranges from 0.70 to 0.90 . Test-retest stability of the scales during a 2-week period ranges from $r=0.62$ to 0.91 . The authors have provided some evidence for convergent validity of the MPI scales.

McGill Pain Questionnaire (MPQ). ${ }^{25}$ Part 2 of the MPQ provides 20 categories of verbal descriptors, with 2 to 6 words in each category, from which pain patients can choose to describe their pain. In the present study, a ranked pain rating index (PRI-R) was calculated for each of the sensory, affective, and evaluative components of pain, on the basis of whether patients had chosen words from each category implying greater or lesser degrees of pain. A total PRI was also calculated by adding the PRI from each of the 3 components. Part 4 of the MPQ allows patients to describe their pain at present, at its worst, and at its least. Test-retest reliability for the 3 pain rating scales averaged $70.3 \%$ during intervals ranging from 3 to 7 days..$^{25}$ Despite documented problems with construct validity $^{3,14}$ and with content validity, ${ }^{2 a, 3}$ the MPQ is still the most widely used assessment tool that attempts to quantify different components of pain.

Center for Epidemiological Studies Depression Scale (CES-D). ${ }^{28}$ The CES-D is a 20-item self-report depression inventory that measures depressive symptoms in the general population. Predictive validity of the CES-D for measuring depressive symptoms in patients with chronic pain has been demonstrated by Turk and Okifuji ${ }^{41}$ and also by Geisser et al. ${ }^{11}$ Participants indicated symptom frequency on a Likert scale ranging from 0 (rarely or none of the time) to 3 (most or all of the time). The CES-D has been shown to have reasonable internal consistency (coefficient alpha ranging from 0.84 to 0.90 ) and test-retest reliability (correlations ranging between 0.48 and 0.67 ), as well as convergent validity.

\section{Statistical Analysis}

Hierarchical multiple regression was used to predict CAT scores, with demographic variables and pain severity and chronicity, secondary appraisal, $A R, A E$, and depression scores entered respectively as independent variables in 5 separate steps (Table 1).

In Step 1, age and sex of the participants, self-reported chronicity of pain, and the MPQ Sensory PRI (the sensory aspect of the pain experience) were entered to control for differences in CAT that might be attributable to these variables. In Step 2, scores for the patients' perceived efficacy at controlling or decreasing their pain (CSQ items \#43 and \#44, respectively) and their perceived ability to have control over their lives (Life Control scale of the MPI) were entered as measures of secondary cognitive appraisal to help calculate the amount of variance in CAT scores predicted by affective variables after considering cognitive appraisal.

The AR variables from the AREQ were entered in Step 3 ahead of $A E$ variables, because it was assumed that $A E$ scores are dependent on how successfully affect is regulated. Thus, scores for Externalizing Defenses, Avoidant Defenses, and Reality-Focused Coping were entered at this stage, whereas scores for Socialized Negative Affect, Positive Affect, and Intense Negative Affect were entered in Step 4. Depressive symptoms, as measured by the CES-D, ${ }^{28}$ were entered in the last step to examine whether the CES-D contributes any significant variance in predicting CAT beyond what is predicted by AREQ variables. 
Table 1. Summary of Hierarchical Multiple Regression Analysis

\begin{tabular}{lcccrc}
\hline \multicolumn{1}{c}{ SteP \& VARIABLES ENTERED } & ADJUSTED & $R^{2}$ & $\begin{array}{c}F \\
R^{2}\end{array}$ & CHANGE & $\begin{array}{c}\text { SIGNIFICANCE OF } \\
\text { CHANGE CHANGE }\end{array}$ \\
\hline 1. Age, sex, PRI-S, pain chronicity & .180 & .147 & .147 & 5.441 & .001 \\
2. CSQ items 43 \& 44, MPI Life Control & .441 & .400 & .253 & 14.887 & .000 \\
3. AR dimensions (EXD, AVD, RFC) & .506 & .453 & .053 & 4.119 & .009 \\
4. AE dimensions (SNA, PA, INA) & .584 & .523 & .070 & 5.575 & .001 \\
5. CES-D & .605 & .542 & .019 & 4.757 & .032 \\
\hline
\end{tabular}

Abbreviations: AR, affect regulation; AE, affective experience; EXD, Extemalizing Defenses; AVD, Avoidant Defenses; RFC, Reality-Focused Coping; PA, Positive Affect; SNA, Socialized Negative Affect; INA, Intense Negative Affect; PRI-S, Pain Rating Index (Sensory); CSQ, Coping Strategies Questionnaire; MPI, Multidimensional Pain Inventory; CES-D, Center for Epidemiological Studies Depression scale. $P<.01$.

Because it was expected that the secondary appraisal variables and some of the AREQ affective variables would be quite highly correlated and would therefore share a great deal of variance in predicting CAT, a second regression analysis was also done, in which the $A R$ and $A E$ variables were entered ahead of the secondary appraisal variables. This allowed us to determine the variance in predicting CAT that is unique to appraisal variables, after accounting for affective variables. Thus, the 2 regression analyses determined the unique variance attributable to both cognitive appraisal and affective variables, from which a calculation of variance shared by affective and appraisal variables was made.

\section{Results}

Demographic information indicated that $62 \%$ of the sample had experienced their pain for more than 5 years, whereas $83 \%$ of the sample had been experiencing pain for more than 2 years. The participants reported a mean score on the CES-D of 21 out of a maximum score of 60 , which was above the cutoff scores of 16 and 19 suggested by both Radloff ${ }^{28}$ and Turk and Okifuji ${ }^{41}$ for detecting significant depressive symptomatology. The mean VAS pain severity rating for all participants was 59.4 on a $100-\mathrm{mm}$ scale, whereas the mean MPQ Total PRI score was 31.5 out of a maximum score of 77 .

Interrater reliability and test-retest reliability of the AREQ were both assessed in this study as described previously. An interrater reliability coefficient of $r=.75$ and a test-retest reliability coefficient of $r=.83$ were obtained, indicating that the AREQ had good interrater reliability and very good test-retest reliability in this study.

The type I error level for the overall regression analysis was set at $\alpha=.05$. With a Bonferroni correction, the type I error level for each step of the regression analysis was $\alpha$ $=.01$. Variables entered in the first 4 steps of both regression analyses resulted in significant changes in adjusted $R^{2}$ values (Table 1). The $R^{2}$ change resulting from entering the CES-D scores into the regression equation in the final step was not significant at $P$ less than .01. The total amount of variance in CAT scores accounted for by all independent variables, after adjusting for sample size, was $54.2 \%$. Demographic variables, pain chronicity, and MPQ Sensory pain ratings together accounted for
$14.7 \%$ of the variance in CAT scores. The secondary appraisal variable set and the 6 affective variables from the AREQ contributed an additional $37.6 \%$ of the variance. After considering secondary appraisal and AREQ variables, CES-D scores contributed $1.9 \%$ of the variance in predicting CAT. The appraisal, AREQ, and CES-D variables accounted for a total of $39.5 \%$ of the adjusted variance in predicting CAT in both analyses.

The significance levels of individual variables in the first regression equation are presented in Table 2 . Relationships were considered significant if probability levels associated with the $t$ statistic were less than $P$ equals .01 . Within the variable set entered in Step 1, lower participant age and higher pain reports on the MPQ-Sensory PRI were related to increased CAT scores (Table 2). For the set of cognitive appraisal variables entered in Step 2 of the regression equation, both CSQ item \#43 (perceived ability to control pain) and the MPI Life Control scale (perceived ability to control one's life) were significant predictors of CAT scores.

Of the 3 AR variables entered in Step 3 of the regression analysis, Reality-Focused Coping was probably the most significant predictor of CAT scores. Although it was associated with a value of $p$ equals .027 , the significance level of this step in the regression analysis was $P$ equals .009 . This suggests that the other 2 variables entered in this step (Avoidant Defenses and Externalizing Defenses) might have been acting as suppressor variables on Reality-Focused Coping in this instance, and that Reality-Focused Coping is likely the variable responsible for the overall significance of this step in the regression analysis.

Of the $3 \mathrm{AE}$ variables entered in Step 4, both Positive Affect and Intense Negative Affect were significant predictors of CAT. The CES-D scores entered in Step 5 of the regression analysis were not a significant predictor of CAT scores after controlling for demographic variables, cognitive appraisal, AR and AE of the patients. The significance levels of individual variables in the second regression equation are not presented in Table 2, because there are very few notable differences. The same variables that obtained individual significance in the first analysis were also significant in the second analysis, with the exception of the MPI Life Control Scale. This variable was not significant in the second analysis $(t=-.274, P=$ 
Table 2. $\boldsymbol{\beta}$ Coefficients and Significance of Individual Variables

\begin{tabular}{|c|c|c|c|c|}
\hline \multirow{2}{*}{$\begin{array}{c}\text { STEP \& VARIABLES } \\
\text { ENTERED }\end{array}$} & \multicolumn{3}{|c|}{ STANDARDIZED } & \multirow{2}{*}{$\begin{array}{c}\text { SIGNIFICANCE OF } \\
t\end{array}$} \\
\hline & в & в & $t$ & \\
\hline 1. Age & -.162 & -.202 & -2.108 & .038 \\
\hline Sex & -.337 & -.011 & -.118 & .906 \\
\hline Pain chronicity & .102 & .010 & .106 & .916 \\
\hline PRI-S & .452 & .372 & 4.059 & .000 \\
\hline $\begin{array}{l}\text { 2. CSQ Control Pain } \\
(\# 43)\end{array}$ & -1.486 & -.254 & -2.619 & .010 \\
\hline $\begin{array}{l}\text { CSQ Decrease Pain } \\
(\# 44)\end{array}$ & -.558 & -.080 & -.839 & .403 \\
\hline MPI Life Control & -1.998 & -.308 & -3.020 & .003 \\
\hline 3. EXD & -.419 & -.066 & -.817 & .416 \\
\hline AVD & -.594 & -.107 & -1.209 & .230 \\
\hline RFC & -1.365 & -.224 & -2.250 & .027 \\
\hline 4. SNA & -.308 & -.036 & -.416 & .679 \\
\hline PA & -1.453 & -.302 & -2.733 & .008 \\
\hline INA & 1.174 & .230 & 2.124 & .036 \\
\hline 5. CES-D & .202 & .298 & 2.181 & .032 \\
\hline
\end{tabular}

Abbreviations: AR, affect regulation; AE, affective experience; EXD, Extemalizing Defenses; AVD, Avoidant Defenses; RFC, Reality-Focused Coping; PA, Positive Affect; SNA, Socialized Negative Affect; INA, intense Negative Affect; PRI-S, Pain Rating Index (Sensory); CSQ, Coping Strategies Questionnaire; MPI, Multidimensional Pain Inventory; CES-D, Center for Epidemiological Studies Depression scale. $P<.01$.

.785), because it was so highly correlated with the $A R$, $\mathrm{AE}$, and CSQ appraisal variables that were entered ahead of it in the regression equation, as shown in Table 3.

The first order correlations between variables in the regression analysis are presented in Table 3. CAT, cognitive appraisal variables, and affective variables are generally quite highly correlated and will therefore share a considerable amount of variance. Consequently, the variance attributable to the first variables entered into the equation will include both unique variance and variance that is shared with subsequently entered variables, whereas variables entered later in the analysis will include only variance that is unique to those variables.

The second variation of the multiple regression analysis was performed to calculate the variance that was shared between the affective and cognitive appraisal variables (Table 1). The unique variance in CAT scores attributable to cognitive appraisal variables is found in the results for the second analysis $\left(R^{2}\right.$ change, .062), whereas the unique variance attributable to $A R$ and $A E$ variables is found in the first analysis $\left(R^{2}\right.$ change, $.053+$ $.070=.123)$. The total variance attributable to both AREQ and cognitive appraisal variables in both analyses was $R^{2}=.376$. Subtracting the unique AREQ variance and unique appraisal variance from the total variance resulted in shared variance of $R^{2}=.191$ between the AREQ and cognitive appraisal variables.

The cognitive appraisal variable set accounted for about $6 \%$ of the unique variance in predicting CAT in the pain patients (Fig 1). The variance in CAT scores ac-

Table 3. First-order Correlations Between Variables in the Regression Equation

\begin{tabular}{|c|c|c|c|c|c|c|c|c|c|c|}
\hline & CSQ 43 & CSQ 44 & $\begin{array}{c}\text { MPI } \\
\text { LIFE } \\
\text { CONTROL }\end{array}$ & $R F C$ & EXD & $A V D$ & SNA & $P A$ & INA & $C E S-D$ \\
\hline CAT & $-.515^{\star}$ & $-.419 *$ & $-.479 *$ & $-.567^{\star}$ & $-.269 *$ & $-.312^{*}$ & -.101 & $-.609 *$ & $.579 *$ & $.685^{\star}$ \\
\hline CSQ Control Pain (43) & & $.493^{*}$ & $.557^{\star}$ & $.441 *$ & .095 & .120 & -.063 & $.396 *$ & $-.297^{\star}$ & $-.461 *$ \\
\hline CSQ Decrease Pain (44) & & & $.544^{\star}$ & $.340 *$ & .138 & .061 & -.084 & $.319 *$ & -.177 & $-.378^{*}$ \\
\hline MPI Life Control & & & & $.543^{*}$ & $.305^{*}$ & .197 & -.018 & $.553^{*}$ & $-.481 *$ & $-.727 *$ \\
\hline RFC & & & & & $.352 *$ & $.416^{*}$ & $.317^{*}$ & $.726^{*}$ & $-.628^{*}$ & $-.712^{*}$ \\
\hline EXD & & & & & & .195 & $-.266^{\star}$ & .177 & -.227 & $-.289 *$ \\
\hline AVD & & & & & & & .128 & .241 & $-.572 *$ & $-.384^{\star}$ \\
\hline SNA & & & & & & & & .226 & -.200 & -.193 \\
\hline PA & & & & & & & & & $-.590 *$ & $-.742 *$ \\
\hline INA & & & & & & & & & & $.675^{*}$ \\
\hline
\end{tabular}

Abbreviations: AR, affect regulation; AE, affective experience; EXD, Extemalizing Defenses; AVD, Avoidant Defenses; RFC, Reality-Focused Coping; PA, Positive Affect; SNA, Socialized Negative Affect; INA, Intense Negative Affect; PRI-S, Pain Rating Index (Sensory); CSQ, Coping Strategies Questionnaire; MPI, Multidimensional Pain Inventory; CES-D, Center for Epidemiological Studies Depression scale.

$* P<.01$, two tailed. 


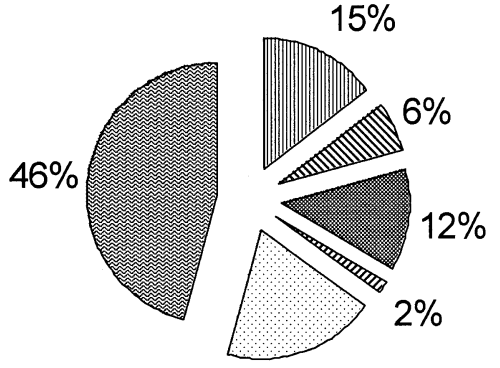

$19 \%$
四 Demographics \& Pain

$\mathbb{N}$ Appraisal

AREQ

CES-D

$\square$ Affect+Appraisal

\section{图 Other}

Figure 1. Variance accounted for by variable sets in the multiple regression analyses.

counted for by all 6 AREQ variables was $12 \%$, indicating that the data obtained from our interview and emotional evaluation of the patients explained twice the amount of unique variance in CAT as did the cognitive appraisal variables. The CES-D contributed $2 \%$ further prediction of CAT scores beyond cognitive appraisal and AREQ variables. The most notable aspect of the data in Fig 1 is that $19 \%$ of the variance in explaining CAT was variance that was shared between the cognitive appraisal and AREQ variables.

\section{Discussion}

This study completed a comprehensive affective assessment of a heterogeneous sample of patients with chronic pain to examine the nature of catastrophizing in those individuals. The results demonstrated that CAT is a complex construct that is strongly associated with cognitive appraisal, AR, and AE. Whereas previous research had emphasized the cognitive and behavioral aspects of CAT, this study has provided evidence for affective variance in CAT in addition to cognitive variance, as well as variance due to a shared cognitive/affective component that is not separable into unique cognitive or affective components. The results highlight the need for considering and accounting for the emotional responses of chronic pain patients in research, rather than excluding or controlling for variance attributable to affective variables. Furthermore, the results also highlight the need to consider the emotional responses of patients and to design assessment protocols and treatment interventions that address $A E$ and $A R$ in addition to the cognitive and behavioral aspects of coping with chronic pain.

The results of this study have increased our understanding of CAT and have clarified some apparent contradictions in the literature. First, it confirms the importance of negative beliefs when patients are catastrophizing. 2,9,10,15,40 However, we support the suggestion by Thorn et $\mathrm{al}^{40}$ that, although those pain-related beliefs are important to CAT, they might be better described as secondary appraisal within the Folkman and Lazarus transactional model of coping. This study confirmed the role of CAT as a cognitive construct by showing that there is significant unique prediction in CAT from patient beliefs about their ability to control their pain or to control their lives in general, when the effects of affective variables are controlled. However, the results also confirmed significant correlations between CAT and different types of negative affect and went a step further by showing that both $A R$ and $A E$ variables were significant predictors of CAT after controlling for the variance due to secondary cognitive appraisal.

It is interesting to note that the AREQ's Reality-Focused Coping scale was the only significant AR variable contributing to prediction of CAT. This supports the proposal of Keefe et al $^{17}$ that CAT might be a maladaptive cognitive coping strategy but provides some further insights into the nature of coping with chronic pain. For some patients, choosing to cope individually on their daily tasks might be an adaptive pain management strategy. However, it was also apparent to the interviewer that a combination of task persistence and avoidance was being used successfully by some patients to avoid negative affect, whereas other patients were unsuccessful in using that same individualistic style of coping.

The clinical observations noted above support the labeling by Westen et $\mathrm{al}^{49}$ of the Reality-Focused Coping scale as an AR strategy versus as a strictly cognitive/behavioral phenomenon. The significant first order correlation between Reality-Focused Coping and Avoidant Defenses suggests that Reality-Focused Coping is also a form of AR that is closely related to avoidant defenses. Further analysis of the varying role of Avoidant Defenses and Reality-Focused Coping in different groups of patients by using profile analysis or cluster analysis might be useful in identifying profiles or clusters of pain patients in whom avoidance and task persistence have been successful, as well as those in whom they have been unsuccessful in decreasing negative affect.

Both the Positive Affect and Intense Negative Affect scales from the AREQ were significant predictors of CAT scores when the $3 \mathrm{AE}$ variables were entered into the regression analyses. The Socialized Negative Affect scale did not appear to have good internal consistency in this sample of pain patients and therefore was not expected to be a significant predictor of CAT. Results indicated that patients who reported less positive affect in their lives tended to report higher levels of CAT. Furthermore, patients who reported more intense negative affect on the Intense Negative Affect scale also appeared to report more CAT, which suggests that CAT is characterized not only by its lack of positive affective quality but also by the intensity of negative affect that is experienced.

It is also interesting that depressive symptoms, as measured by the CES-D, did not contribute significant variance to prediction of CAT after controlling for cognitive appraisal, $A E$, and $A R$ variable sets. This suggests that after we consider patients' beliefs in their ability to control pain and their lives and both the $A E$ and $A R$ variables, there is an insignificant portion of the remaining variance in CAT scores that is related to depressive symptomatology. Thus, the significant relationship demonstrated in previous research between depressive symptoms and $\mathrm{CAT}^{35}$ has been accounted for by the significant relationships between variables used in the comprehensive assessment of $A E$ and $A R$ in this study. 
Although patient age and pain severity were entered in the first step of the regression analysis to control for the effects of these variables, their effect on prediction of CAT scores is worthy of further discussion. The results suggest that people with more severe pain could be more susceptible to thinking in catastrophic terms about their pain symptoms. However, although the prediction in CAT by age was not significant at the $P$ less than .01 level, the results of this study showed a tendency toward prediction of CAT scores by age $(P=.038)$. These results might not be as easily explained as those for level of pain severity. Decreased CAT could be expected with increased age if older patients have learned more adaptive ways of coping with their pain over the years. Or, an analysis of the ages of diagnostic groups of patients in this study showed that the groups containing arthritis patients tended to be older. Because patients with arthritis have a medical explanation for their symptoms and because they have some treatment choices available to them, these patients might have perceived themselves to have more control over their symptoms than patients with FMS or TMD (which are less amenable to medical treatment), resulting in less tendency to catastrophize. However, there were no significant differences in CAT scores between different diagnostic groups, suggesting that this effect is largely due to age. Thus, although the multiple regression analyses in this study suggested that CAT is composed of significant cognitive appraisal and affective components after controlling for patient age, it should be noted that there was a trend for CAT to be more pronounced in younger patients.

One of the most significant findings in this study is the significant amount of variance shared by secondary appraisal and affective variables in predicting CAT, which is not attributable to shared method variance. This cognitive/affective overlap is significant because it suggests that there is a combined cognitive/affective dimension to CAT that defies our efforts to completely separate the effects of either the cognitive or affective components of CAT. On a theoretical level, this finding is consistent with the fact that we often have feelings about what we are thinking and often think about what we are feeling, and those thoughts and feelings are often linked together and difficult to separate. At a clinical level, this cognitive/ affective overlap helps to explain why interventions such as cognitive restructuring training can be very difficult for some patients. Those patients frequently have a great deal of negative affect that is tied up with their cognitions. They might be heavily defended against acknowledging or identifying their negative feelings, making it very difficult for them to identify the core beliefs that underlie their cognitions and making it difficult to challenge their habitual negative patterns of thinking. For those patients, improved assessment of $A R$ and $A E$ and interventions such as individual or group psychotherapy might be advisable to help them improve their ability to express and identify feelings, establish the links to their negative thoughts, and better enable them to challenge the negative thoughts and beliefs that might be hindering their attempts to cope.
Matching specific forms of intervention to specific affective profiles would allow clinicians to target specific styles of coping or AR for improvement and would potentially reduce or make more efficient use of the time spent on providing treatment components to patients that might be less necessary for each individual patient. For example, patients who tend to use avoidance to distract themselves from pain symptoms and to focus on reality-based coping styles, which is clearly adaptive in many respects, might have a difficult time relaxing or pacing their daily activities. For this group, it would be more appropriate to focus our interventions on pain management skills such as pacing and various forms of relaxation training. On the other hand, patients who avoid acknowledging the emotions in their lives might have a difficult time identifying the negative feelings and thoughts that often accompany their pain experience. The use of avoidance in this group is often less adaptive for coping with chronic pain, and this group might require more intensive group or individual psychotherapy to help them acknowledge and identify their emotions and to learn cognitive restructuring techniques.

When possible differences in subgroups of pain patients are considered together with the communal model of CAT described by Sullivan et $\mathrm{al}^{38}$ and the significant relationships between affective variables and CAT reported in this study, we suggest that affective variables could possibly act as a link between cognitive factors, communal/behavioral factors, and CAT in explaining pain outcomes. A potential direction for research in this area would be to study whether AE and habitual styles of AR mediate the relationship between cognitive variables, individual differences in communal versus individual styles of coping, and tendency to catastrophize in different subgroups of pain patients.

There are some potential limitations to the present study that could limit generalizability of the results and that suggest possible avenues for future research. First, the disproportionate number of women in this study potentially limits generalizing the results to male patients. However, men and women differed significantly only on the relative degree to which they used avoidant defenses to regulate negative affect, suggesting that men and women in the study were generally very similar and that the gender makeup of the research sample had little effect on the overall results.

Second, even though the clinical interviews attempted to rate patients on habitual AR strategies they have developed during their lifetime, the study was cross-sectional in nature. Future research should follow changes in $A E$ and $A R$ over time, perhaps as part of a longitudinal outcome study or an evaluation study of different treatment interventions for patients who tend to catastrophize. Longitudinal research designed to follow affective, cognitive, and coping variables as well as outcome measures over time would help to develop causal models showing the influence of multiple variables on pain outcome measures. 
It could be argued that the use of such a heterogeneous sample in this study could compromise the internal validity of the study. For example, it could be argued that the results might not apply to all diagnostic subgroups of pain patients, because each subgroup's pain experience could be qualitatively different. Another potential pitfall that could compromise internal validity is the increased possibility of random error variance from studying less homogeneous diagnostic groups. In response to these arguments, however, it should be noted that $46 \%$ of the patients in this study with a confirmed pain diagnosis had multiple diagnoses. It was our feeling that including these patients in the present study would increase the generalizability of the results and increase the external validity of the study. There is often a tradeoff between attempting to maximize either internal or external validity. A conscious attempt was made in this study to increase the generalizability of the results, but it is recognized that internal validity could potentially have been compromised in the process.

A potential methodologic concern in this study is the possibility that the raters who coded affective data from the interviews by using the Q-sort procedure could potentially have been biased in their coding by their knowledge of the study's hypothesis. The primary safeguard against this concern is that all variables in the study other than the $A E$ and $A R$ variables were measured by using the participants' self-report responses to questionnaires.

\section{References}

1. Affleck $G$, Tennen $H$, Urrows $S$, Higgins P: Neuroticism and the pain-mood relation in rheumatoid arthritis: Insights from a prospective daily study. J Consul Clin Psychol 60:119126,1992

2. Boothby JL, Thorn BE, Stroud MW, Jensen MP: Coping with pain. In: Gatchel RJ, Turk DC (eds): Psychosocial Factors in Pain: Critical Perspectives. New York, NY, The Guilford Press, 1999, pp. 343-359

2a. Costa PT, Fernandez E, Kerns RD, Papciak AS: New directions in psychological testing in chronic pain. Paper presented at: American Pain Society 17th Annual Meeting, 1998, San Diego, CA.

3. Crockett DJ, Prkachin KM, Craig KD, Greenstein H: Social influences on factored dimensions of the McGill Pain Questionnaire. J Psychosom Res 30:461-469, 1986

4. Crombez G, Vlaeyen JWS, Heuts PHTG, Lysens R: Painrelated fear is more disabling than pain itself: Evidence on the role of pain-related fear in chronic back pain disability. Pain 80:329-339, 1999

5. Davey GCL, Jubb M, Cameron C: Catastrophic worrying as a function of changes in problem-solving confidence. Cognit Ther Res 20:333-344, 1996

6. Davey GCL, Levy S: Internal statements associated with catastrophic worrying. Personality \& Individual Differences 26:21-32, 1999

7. Geisser ME, Robinson ME, Henson CD: The Coping Strate-
The questionnaires were not scored and the statistical analyses were not completed until after all interviews were conducted and the Q-sorts were completed. Thus, the raters were blinded to the values of all variables for each subject, including scores for the affective variables from other participants, which were calculated after all interviews and Q-Sorts were completed.

In summary, the results of this study paint a picture of catastrophizing in patients with chronic pain that is more consistent with the images we have of people who have experienced catastrophic events in their lives. Catastrophizing appears to be a complex combination of perceived lack of control, lack of reality-focused coping abilities, lack of positive affect, and presence of intense negative affect. The results have implications for designing assessment protocols and treatment interventions for helping patients with chronic pain cope with their symptoms, because they suggest that interventions and assessment should focus on emotional responses as well as beliefs, cognitive appraisals, and behavioral coping strategies.

\section{Acknowledgments}

This article represents a portion of the doctoral dissertation research conducted by David A. Jones in partial fulfillment of the requirements for the Doctor of Philosophy degree at the University of Western Ontario.

gies Questionnaire and chronic pain adjustment: A conceptual and empirical reanalysis. Clin J Pain 10:98-106, 1994

8. Geisser ME, Robinson ME, Keefe FJ, Weiner ML: Catastrophizing, depression and the sensory, affective and evaluative aspects of chronic pain. Pain 59:79-83, 1994

9. Geisser ME, Robinson ME, Riley JL: Pain beliefs, coping, and adjustment to chronic pain: Let's focus more on the negative. Pain Forum 8:161-168, 1999

10. Geisser ME, Roth RS: Knowledge of and agreement with chronic pain diagnosis: Relation to affective distress, pain beliefs and coping, pain intensity, and disability. J Occup Rehabil 8:73-88, 1998

11. Geisser ME, Roth RS, Robinson ME: Assessing depression among persons with chronic pain using the Center for Epidemiological Studies-Depression Scale and the Beck Depression Inventory: A comparative analysis. Clin J Pain 13:163170, 1997

12. Härkäpää K: Relationships of psychological distress and health locus of control beliefs with the use of cognitive and behavioral coping strategies in back pain patients. Clin J Pain 7:275-282, 1991

13. Hill A, Niven CA, Knussen C: The role of coping in adjustment to phantom limb pain. Pain 62:79-86, 1995

14. Holroyd KA, Holm JE, Keefe FJ, Turner JA, Bradley LA, Murphy WD, Johnson P, Anderson K, Hinkle AL, O'Malley WB: A multi-center evaluation of the McGill Pain Questionnaire: Results from more than 1700 chronic pain patients. Pain 48:301-311, 1992 
15. Jensen MP, Turner JA, Romano JM: Chronic pain coping measures: Individual vs composite scores. Pain 51:273-280, 1992

16. Keefe FJ, Kashikar-Zuck S, Robinson E, Salley A, Beuapre P, Caldwell D, Baucom D, Haythornthwaite J: Pain coping strategies that predict patients' and spouses' ratings of patients' self-efficacy. Pain 73:191-199, 1997

17. Keefe FJ, Lefebvre JC, Smith SJ: Catastrophizing research. Pain Forum 8:176-180, 1999

18. Kerns RD, Turk DC, Rudy TE: The West Haven-Yale Multidimensional Pain Inventory (WHYMPI). Pain 23:345-356, 1985

19. Lazarus RS, Folkman S: Stress, Appraisal, and Coping. New York, NY, Springer, 1984

20. Lefebvre JC, Lester N, Keefe FJ: Pain in young adults. II: The use and perceived effectiveness of pain-coping strategies. Clin J Pain 11:36-44, 1995

21. Main CJ, Waddell G: A comparison of cognitive measures in low back pain: Statistical structure and clinical validity at initial assessment. Pain 46:287-298, 1991

22. Martin MY, Bradley LA, Alexander RW, Alarcon GS, Triana-Alexander M, Aaron LA, Alberts KR: Coping strategies predict disability in patients with primary fibromyalgia. Pain 68:45-53, 1996

22a. Martin MY, Bradley L: Catastrophizing: Effect on pain and function. Paper presented at: American Pain Society 18th Annual Meeting, 1999, Fort Lauderdale, FL.

23. McCracken LM, Goetsch VL, Semenchuk EM: Coping with pain produced by physical activity in persons with chronic low back pain: Immediate assessment following a specific pain event. Behav Med 24:29-34, 1998

24. McDermid AJ, Rollman GB, McCain GA: Generalized hypervigilance in fibromyalgia: Evidence of perceptual amplification. Pain 66:133-144, 1996

25. Melzack R: The McGill Pain Questionnaire: Major properties and scoring methods. Pain 1:277-299, 1975

26. Osman A, Barrios FX, Kopper BA, Hauptmann W, Jones J, O'Neill E: Factor structure, reliability, and validity of the Pain Catastrophizing Scale. J Behav Med 20:589-605, 1997

27. Parker JC, Wright GE: The implications of depression for pain and disability in rheumatoid arthritis. Arthritis Care Res 8:279-283, 1995

28. Radloff LS: The CES-D scale: A self-report depression scale for research in general populations. Applied Psychological Measurement 1:385-401, 1977

29. Riley JL III, Robinson ME, Geisser ME: Empirical subgroups of the Coping Strategies Questionnaire-Revised: A multisample study. Clin J Pain 15:111-116, 1999

30. Robinson ME, Riley JL 3rd, Myers CD, Sadler IJ, Kvaal SA, Geisser ME, Keefe FJ: The Coping Strategies Questionnaire: A large sample, item level factor analysis. Clin J Pain 13:4349, 1997

31. Robinson ME, Riley JL III: The role of emotion in pain, in Gatchel RJ, Turk DC (eds): Psychosocial Factors in Pain: Critical Perspectives. New York, NY, The Guilford Press, 1999, pp 74-88

32. Rosenstiel AK, Keefe FJ: The use of coping strategies in chronic low back pain patients: Relationship to patient characteristics and current adjustment. Pain 17:33-44, 1983

33. Rudy TE, Turk DC, Zaki HS, Curtin HD: An empirical taxometric alternative to traditional classification of temporomandibular disorders. Pain 36:311-320, 1989

34. Shutty MS, DeGood DE: Cluster analyses of responses of low-back pain patients to the SCL-90: Comparison of empirical versus rationally derived subscales. Rehabil Psychol 32: 133-144, 1987

35. Sullivan MJ, D'Eon JL: Relation between catastrophizing and depression in chronic pain patients. J Abnorm Psychol 99:260-263, 1990

36. Sullivan MJL, Bishop SR, Pivik J: The Pain Catastrophizing Scale: Development and validation. Psychol Assess 7:524-532, 1995

37. Sullivan MJL, Stanish W, Waite H, Sullivan M, Tripp DA: Catastrophizing, pain, and disability in patient with softtissue injuries. Pain 77:253-260, 1998

38. Sullivan MJL, Thorn B, Haythornthwaite JA, Keefe $F$ Martin M, Bradley LA, Lefebvre JC: Theoretical perspectives on the relation between catastrophizing and pain. Clin J Pain 17:52-64, 2001

39. Swartzman LC, Gwadry FG, Shapiro AP, Teasell RW: The factor structure of the Coping Strategies Questionnaire. Pain 57:311-316, 1994

40. Thorn BE, Rich MA, Boothby JL: Pain beliefs and coping attempts. Pain Forum 8:169-171, 1999

41. Turk DC, Okifuji A: Detecting depression in chronic pain patients: Adequacy of self-reports. Behav Res Ther 32:9-16, 1994

42. Turk DC, Rudy TE: Towards a comprehensive assessment of chronic pain patients. Behav Res Ther 25:237-249, 1987

43. Turk DC, Rudy TE: Toward an empirically derived taxonomy of chronic pain patients: Integration of psychological assessment data. J Consult Clin Psychol 56:233-238, 1988

44. Turner JA, Jensen MP, Romano JM: Do beliefs, coping, and catastrophizing independently predict functioning in patients with chronic pain? Pain 85:115-125, 2000

45. Tuttle DH, Shutty MS, DeGood DE: Empirical dimensions of coping in chronic pain patients: A factorial analysis. Rehabil Psychol 36:179-188, 1991

46. Ulmer JF: An exploratory study of pain, coping, and depressed mood following. J Pain Symptom Manage 13:148 157, 1997

47. Vlaeyen JW, Linton SJ: Fear-avoidance and its consequences in chronic musculoskeletal pain: A state of the art. Pain 85:317-332, 2000

48. Watson D, Pennebaker JW: Health complaints, stress, and distress: Exploring the central role of negative affectivity. Psychol Rev 96:234-254, 1989

49. Westen D, Muderrisoglu S, Fowler C, Shedler J, Koren D: Affect regulation and affective experience: Individual differences, group differences, and measurement using a Qsort procedure. J Consult Clin Psychol 65:429-439, 1997

50. White KP, Speechley M, Harth M, Ostbye T, : The London Fibromyalgia Study: the prevalence of fibromyalgia in London, Ontario. J Rheumatol 1999:26(7):1570-1576. 